\title{
EMBEDDED MULTIPLE DESCRIPTION CODING FOR PROGRESSIVE IMAGE TRANSMISSION OVER UNRELIABLE CHANNELS
}

\author{
IRISA-INRIA \\ Campus de Beaulieu \\ 35042 Rennes cedex \\ France \\ Thomas.Guionnet@irisa.fr
}

Thomas Guionnet, Christine Guillemot, Stéphane Pateux

\begin{abstract}
A multiple description scalar quantization (MDSQ) based coding system can be regarded as a source coder (quantizer) followed by a channel coder, i.e. the combination of index and codeword assignment. The redundancy, or the correlation between the descriptions, is controlled by the number of diagonals covered by the index assignment. We consider here the usage of multiple description uniform scalar quantization (that we call MDUSQ) for robust and progressive transmission of images over unreliable channels. The progressive feature is an important factor for rate control in non stationary (varying bandwidth) communication environments. In this context, the paper describes an embedded index assignment strategy that provides improved rate-distortion performances in progressive transmission scenarios, against index assignments defined so far for MDSQ. The MDUSQ together with the embedded index assignment algorithm are incorporated into the JPEG2000 verification model. The approach is compared against a progressive multiple description scheme based on a polyphase transform (PT) decomposition of the signal.
\end{abstract}

\section{INTRODUCTION}

Multiple description coding has been introduced as a generalization of source coding subject to a fidelity criterion for communication systems that use diversity to overcome channel impairments. Several correlated coded representations of the signal are created and transmitted on different channels. The encoder and decoder design goals are therefore to achieve the best average ratedistortion performance when all the channels work, subject to constraints on the average distortion when only a subset of channels is received correctly. Extensive work has been dedicated to finding optimal achievable rate-distortion regions [1], [2] and to the design of practical compression systems approaching these theoretical bounds. A number of compression systems based on multiple description transformations [3], [4], [5], or quantization [6], [7] has been proposed. In [6], the Lloyd-Max algorithm is generalized in order to design multiple description quantizers that would approach the optimal performance bounds in the sense of minimum average distortion. The design of MDSQ in [6] assumes fixed length codes and fixed codebook sizes. Significant improvements are achieved in [7] by using variable length codes instead of fixed length codes and by designing the quantizer under the constraint of a given entropy instead of a codebook size. Both approaches above assume that the codebooks are optimized and adapted to the signal statistics. Such adaptive codebook design may lead to an encoding complexity not very realistic for practical communication systems.

We consider instead uniform quantizers followed by entropy coders. Our goal being in addition to allow for rate control in non stationary (or bandwidth varying) communication environments, we consider a progressive and embedded representation of the bitstream with fine grain scalability. The representation of each description, inspired from the EBCOT coding principles [8], relies on a bit-plane encoding. We develop an index assignment which allows to improve the rate-distortion performance against so far proposed index assignments in the context of progressive and embedded bit streams. The technique is compared against a polyphase transform based progressive multiple description approach. Both techniques, the multiple description uniform scalar quantizer, called here MDUSQ, with the corresponding embedded index assignment, and the polyphase transform, are incorporated into the JPEG2000 verification model. Both techniques are applied on the wavelet transform coeffcients. The experiments show that uniform quantization coupled with an appropriate embedded index assignment can lead to successive refinement of information with good rate-distortion performance in multiple channel transmission systems. The approach is therefore well suited for fine grain rate adaptation and for progressive transmission of each description. This approach can outperform the embedded and progressive MDC coding algorithm based on the polyphase transform applied in the wavelet transform domain, for high redundancy rates. However, for low levels of redundancy, better performances have been obtained with the polyphase-transform based algorithm.

\section{PROGRESSIVE MULTIPLE DESCRIPTION UNIFORM SCALAR QUANTIZATION}

The system is composed of a quantizer mapping the sequence of real-valued source samples into a sequence of integer-valued source symbols taking their values in a finite alphabet $C$ composed of $m$ symbols. Each source symbol is then mapped to two sets of components (index) that will be coded with fixed or variable length codes and transmitted over the channels. These two set of indexes take their values in finite alphabets $I$ and $J$ composed respectively of $M_{I}$ and $M_{J}$ symbols. $C$ is a subset of $I \times J$. These mappings are referred to as the index assignments and are defined by a 2 dimension matrix as shown in Fig 1a. This matrix allows to map $m$ numbers into two sets of entries $i=1, \ldots M_{I}$ and $j=1, \ldots M_{J}$. The examples of Fig 1a consider balanced descriptions with in- 
dexes taking their values in the same finite alphabet (alphabet of same size, $M_{I}=M_{J}=2^{q}$ ). Each interval of the central partition is referred to by its coordinates $(i, j)$ in the index assignment.

An MDSQ-based decoder comprises three inverse quantizers which map the received indexes $i$ and $j$ onto reconstruction levels $\hat{x}_{(i, j)}, \hat{x}_{i}$, and $\hat{x}_{j}$, taking values respectively in the codebooks $\hat{X}_{C}=\left\{\hat{x}_{(i, j)},(i, j) \in C\right\}, \hat{X}_{I}=\left\{\hat{x}_{i}, i \in I\right\}$ and $\hat{X}_{J}=\left\{\hat{x}_{j}, j \in J\right\}$. The performance of the whole system is very much dependent on the index assignment. The index assignment, for a given amount of redundancy or correlation, is designed to minimize the spread ${ }^{1}$ of quantization intervals referred to by a given index. The redundancy is controlled by choosing the number of diagonals covered by the index assignment. If only the main diagonal is used, we have maximum correlation (duplication of information).

Considering a bit-plane and progressive encoding of each description, let $b_{i}$ and $b_{j}$ be the number of bitplanes received respectively for each description (for each sequence of indexes). The formulas expressing central and side average granular distortions of i.i.d. Gaussian sources [6] can be applied here to derive the average distortion when receiving $b_{i}$ and $b_{j}$ bit planes respectively on the two channels. The average granular distortion value is then given by

$$
\overline{d_{b_{i} b_{j}}}=\sum_{k=1}^{2^{b_{i}}} \sum_{l=1}^{2^{b_{j}}}\left(\sum_{m=1}^{K_{i}} \sum_{n=1}^{K_{j}} \int_{(\alpha, \beta) \in C} d\left(x, \hat{x}_{k, l}\right) P(x) d x\right),
$$

where $K_{i}=\frac{M_{I}}{2^{b_{i}}}$ and $K_{j}=\frac{M_{J}}{2^{b_{j}}}$, and where $\alpha=K_{i} k+m$ and $\beta=K_{j} l+n$. The bits received on each description provide entries to quadrants and subquadrants of the index assignment matrix as shown in Fig. 1. The reconstruction value $\hat{x}_{k, l}$ is computed as the barycenter of the reconstruction values in the cells addressed by the set of entries given by the different values of $\alpha$ and $\beta$.

(a)
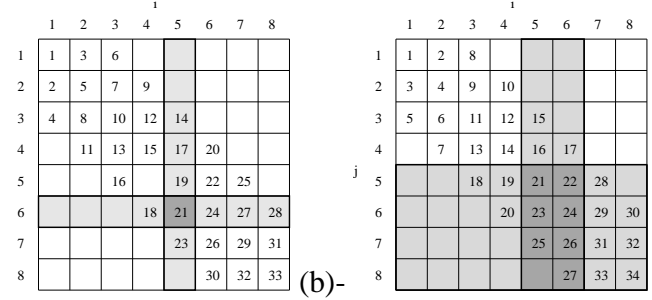

Fig. 1. Index assignments (IA) and cells (in dark grey) considered for reconstruction for (a)- MDSQ and modified linear IA with received indexes $i$ or/and $j$; (b) progressive MDSQ and embedded IA with one and two bit-planes received respectively for indexes $j$ and $i$.

Similarly to [6], one could then, by using a Lloyd-Max algorithm, compute the central quantizer as well as the reconstruction values $\hat{x}_{k, l}$ so that the average distortion $\overline{d_{b_{i} b_{j}}}$ is minimized for different combinations of the values of the numbers of bit planes $b_{i}$ and $b_{j}$. However, such a signal dependent optimization is rather complex, and in addition optimal quantizers require the transmission of the codebooks. We consider instead using a uniform quantizer (MDUSQ) jointly with a bit-plane based statistical encod-

\footnotetext{
${ }^{1}$ The spread is defined as the difference between the maximum and minimum values of the central quantizer interval indexes, in each projection (horizontal and vertical) of the matrix.
}

ing. Fig.(2) shows comparative rate-distortion performance between the MDSQ and the MDUSQ for an i.i.d Gaussian source of mean 0 and unit variance.

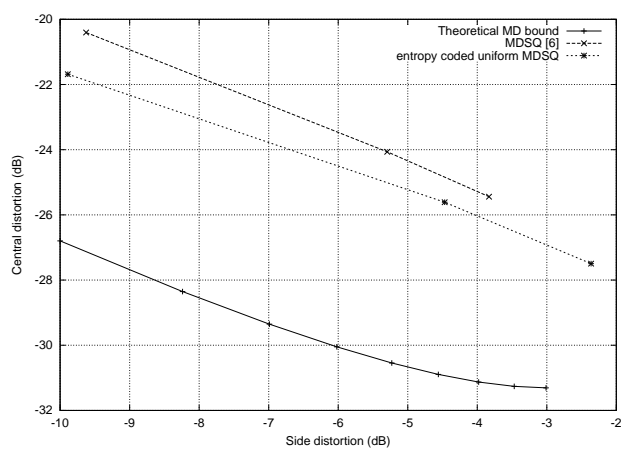

Fig. 2. Comparative rate-distortion performance between the MDSQ and the MDUSQ coding for an i.i.d Gaussian source (2.6 bpss/description).

Adopting the modified linear index assignment proposed in [6] with $M_{I}=M_{J}=8$ and 5 diagonals covered, Fig.(3) shows the practically observed and theoretical central and side granular distortion values in terms of the number of received bit planes for an i.i.d Gaussian source. There is a significant gap between the theoretical distortion-rate bounds variations and the practically observed distortion decrease rate.

\begin{tabular}{|c||c|c|c|c|}
\hline & 0 & 1 & 2 & 3 \\
\hline \hline central & 1.0027 & 0.2570 & 0.1115 & 0.0033 \\
\hline theoretical bound & 1 & 0.0626 & 0.0043 & 0.00031 \\
\hline \hline side 1/2 & 1.0027 & 0.5091 & 0.3496 & 0.2683 \\
\hline theoretical bound & 1 & 0.25 & 0.0625 & 0.0156 \\
\hline
\end{tabular}

Fig. 3. Distortion performances of progressive MDUSQ using the midified linear index assignment [6], and corresponding theoretical bounds [1]. The values are given for different numbers of bit planes received for each description.

The gap can be explained partly from the shape and from the spread of the index assignment. Let us consider the modified linear index assignment shown in Fig.(1-a) and proposed in [6]. If for a sample the two first MSBs for index $i$ are 0 , then the cells labelled $1,2,3,4,5,8,11$ are considered for reconstruction. Therefore, given the shape of the mapping table, the MSB of the second description will be redundant and will not convey any additional information. Similarly, if for a sample the first MSB for index $i$ is 0 , then the value of the MSB for index $\mathrm{j}$ can be predicted and is likely to be 0 . The LSBs carry more information. In other words the MSBs are more redundant than the LSBs. This property may be exploited for improved robustness of the stream. In addition, the modified linear index assignment considered [6] has been designed in order to minimize the spread in lines and in columns. However, in the case of progressive transmission, one has to measure the spread not only in lines and in columns, but on the rectangular shaped areas as shown in Fig.(1-b).

\section{EMBEDDED INDEX ASSIGNMENT}

This observation has motivated the design of a new index assignment strategy better suited for progressive transmission. The index assignment is designed in order to minimize the spread over a square area corresponding to balanced descriptions $\left(b_{i}=b_{j}\right)$. If 
$b_{i}>b_{j}$, we have a central decoding for the first $b_{j}$ bit planes and a side decoding for the remaining $b_{i}-b_{j}$ bits of the description of higher resolution. As in previous work, the amount of redundancy between the two descriptions is adjusted by tuning the number $2 \times d+1$ of diagonals covered by the index assignment. Let $M_{I}=2^{q_{I}}$ and $M_{J}=2^{q_{J}}$ be the sizes of the two index alphabets, and let $d$ be as defined above. The redundancy rate is given by $R_{c}=\frac{R_{e f f}\left(d, q_{I}, q_{J}\right)}{q_{I}+q_{J}}$, where $R_{e f f}\left(d, q_{I}, q_{J}\right)=$ $\log _{2}\left(N\left(d, q_{I}, q_{J}\right)\right)$ and where $N\left(d, q_{I}, q_{J}\right)$ is the number of cells covered by the index assignment.

Let $\left(O_{I}, O_{J}\right)$ be the upper left index pair of the index assignment table $T$ of size $M_{I} \times M_{J}$. The central quantizer cells to be mapped on $T$ are labelled from 1 to $N\left(d, q_{I}, q_{J}\right)$. The labels are considered sequentially. The current label to be mapped on $T$ is indicated by "current", and is initialized to 1 . The embedded index assignment is created using the following recursive function $F$.

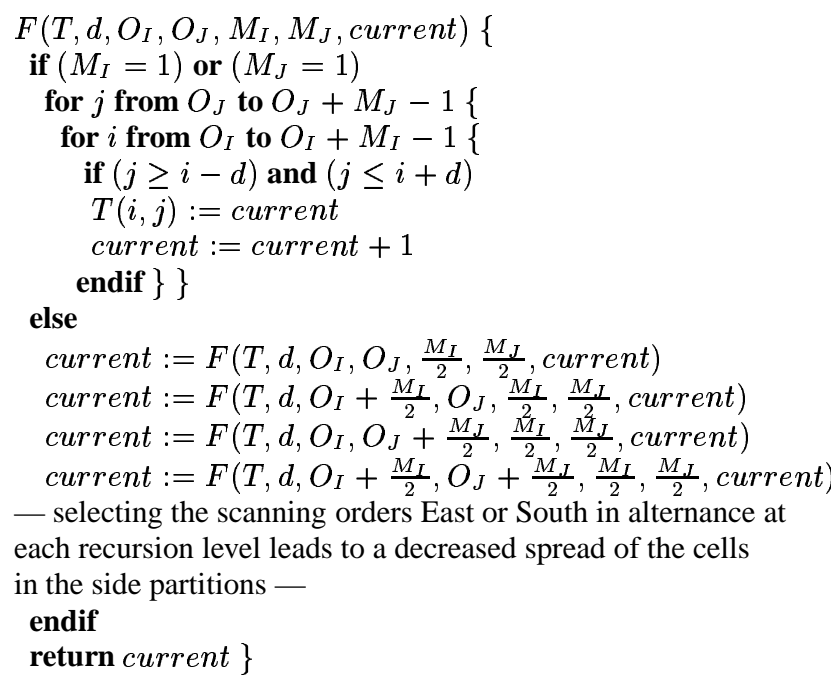

Fig.(1.b) shows an example of embedded index assignment, for $q_{I}=q_{J}=3$ and for $d=2$. The distortion values (MSE) obtained for varying numbers of bit planes received are given in Fig.4. The embedded index turns out to improve significantly the rate-distortion performances of the MDUSQ progressive decoding.

\begin{tabular}{|c||c|c|c|c|}
\hline & 0 & 1 & 2 & 3 \\
\hline \hline central & 1.0027 & 0.1670 & 0.0313 & 0.0031 \\
\hline theoretical bound & 1 & 0.0626 & 0.0044 & 0.00029 \\
\hline \hline side $1 / 2$ & 1.0027 & 0.3645 & 0.2338 & 0.1928 \\
\hline theoretical bound & 1 & 0.25 & 0.0625 & 0.0156 \\
\hline
\end{tabular}

Fig. 4. Distortion performances of progressive MDUSQ using the embedded index assignment, and corresponding theoretical bounds [1]. The values are given for different numbers of bit planes received for each description.

\section{OPTIMAL REDUNDANCY ALLOCATION}

The amount of redundancy between the two descriptions is adjusted by the parameter $d$ for MDSQ. For the polyphase transform, optimal redundancy allocation is derived in [9] as a function of the channel failure probability $p$. Optimal redundancy allocation for the MDSQ is derived in a slightly different way in [3]. Using the results of [3], one can express the parameter $d$ as a function of $p$.
We know from [3] that for $M_{I}=M_{J}=2^{q}$, The central and side distortions $D_{c}$ and $D_{s}$ are given by

$$
\begin{aligned}
& D_{c}=C \sigma^{2} 2^{-2 q\left(1+\frac{1}{n}\right)}, \\
& D_{s}=S \sigma^{2} 2^{-2 q\left(1-\frac{1}{n}\right)},
\end{aligned}
$$

where $d=2^{\frac{q}{n}}, \sigma^{2}$ is the source variance and where $C$ and $S$ are determined by the source pdf and the encoding method. Similarly to [9] one may minimize the cost function $J=D_{c}+\lambda D_{s}$, by having $\left.\frac{\delta J}{\delta n}\right|_{n=n^{*}}=0$ which leads to

$$
\frac{1}{n^{*}}=-\frac{1}{4 q} \log _{2} \frac{\lambda S}{C} \text {. }
$$

The channel failure probability $p$ is introduced by deriving the average distortion

$$
D=(1-p)^{2} D_{c}+2 p(1-p) D_{s}+p^{2} \sigma^{2},
$$

the minimization of which leads to

$$
\begin{gathered}
\frac{1}{n^{*}}=-\frac{1}{4 q} \log _{2} \frac{2 p S}{(1-p) C}, \\
d^{*}=2^{-\frac{1}{4} \log _{2} \frac{2 p S}{(1-p) C}} .
\end{gathered}
$$$$
\text { and }
$$

Notice that for practical implementation, one may have to discretize the diagonal parameter $d$. This can be generalized to transform codes by searching an optimal $d$ for each subband [3].

\section{ENCODING PROCEDURE AND RESULTS}

The two above techniques have been incorporated into the JPEG2000 verification model (VM8.0) [8].The MDUSQ and corresponding index assignment, as well as the polyphase decomposition followed by the selective quantization are applied on the different subbands resulting from the wavelet transform. The polyphase decomposition and the corresponding selective quantization are applied similarly. The EBCOT-based coding algorithm creates a progressive bitstream for blocks of the image. Then all these bitstreams are combined using a rate/distortion optimization in order to generate the overall progressive bitstream for the entire image.

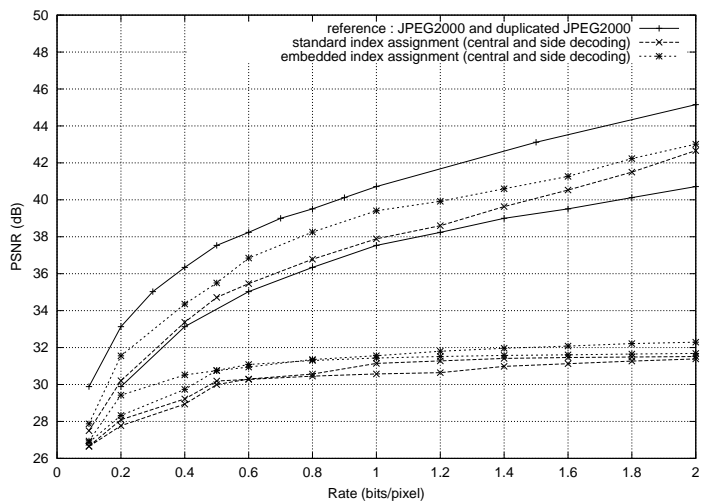

Fig. 5. Comparative side and central rate-distortion performances between the modified linear index assignement and the embedded index assignment (Lena $512 \times 512)$.

Fig. 5 shows that when used jointly with the MDUSQ, in a context of embedded and progressive transmission, the embedded index assignement outperforms the nested index assignement [6]. The results in Fig.5 have been obtained for $d=4$. Fig.6 shows comparative results between the MDUSQ followed by an embedded index assignement (with $d=4$ ) and the progressive approach based on the polyphase decomposition of the different 
subbands (20\% redundancy). Better side PSNR-rate performances have been obtained with the MDUSQ and the embedded index assignment at very low bit rate (below $0.4 \mathrm{bpp}$ ), for comparable central PSNR. However, at higher rates, better side PSNR performances are obtained with the polyphase decomposition followed by a selective quantization.

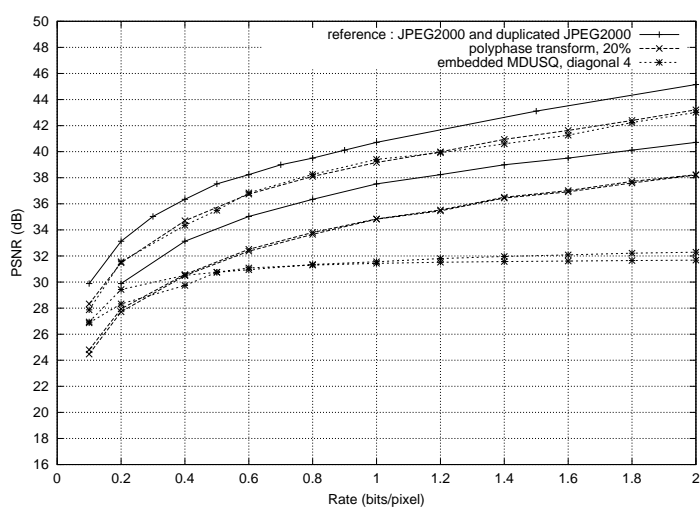

Fig. 6. Comparative side and central rate-distortion performances of the MDUSQ and the approach based on the subband polyphase component decomposition (Lena $512 \times 512$ ).

Fig. 7 provides comparative results at 0.25 and 0.5 bpp per description between the algorithms described above and (1) the solutions based on MDSQ and SPIHT described in [10] and (2) the approach based on a polyphase transform and selective quantization followed by SPIHT proposed in [9]. The respective performances of the different solutions are highly dependent on the targeted overall and redundancy rates. Note that the algorithms described here inherit from the relative lower rate-distortion performances of EBCOT with respect to SPIHT, but also from additional features allowing for spatial scalability as well as progressive and embedded bitstreams.

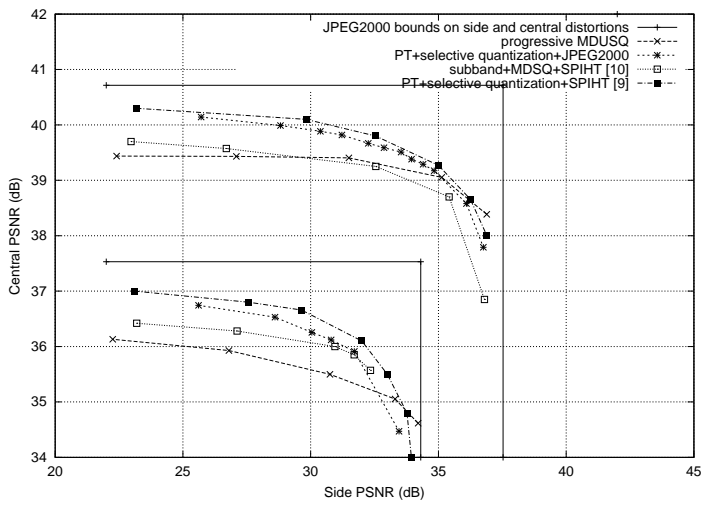

Fig. 7. Comparative central and side PSNR performances of (a) progressive MDUSQ, (b) PT+selective quantization+JPEG2000, (c) Subband+MDSQ+SPIHT [10], (d) PT+selective quantization+SPIHT [9] (Lena $512 \times 512)$.

\section{CONCLUSION}

In this paper, we have presented two embedded multiple description coding systems for progressive image transmission over unreliable channels. The ultimate goal is fine grain media and redundancy rate adaptation on non stationary channels. The first algorithm developped relies on multiple description scalar uniform quantization followed by an embedded index assignement.
This technique has been incorporated in a JPEG2000 encoder, the quantization and index assignement being applied on the different subbands. The resulting index sequences being compressed by using an EBCOT based bit-plane encoding. The experimental results show the benefits of the embedded index assignement versus the strategies published so far, for the particular situation of progressive transmission. The second approach relies on a polyphase component decomposition of the subbands resulting from the wavelet transform followed by a selective quantization of the different polyphase components. Overall, both multiple descriptions approaches are well suited for progressive transmission. The respective performances of the two solutions are highly dependent on the targeted bit rates and amounts of redundancy. At low bit rates, and for high amounts of redundancy, the progressive MDUSQ outperforms the approach based on the polyphase transform and selective quantization. Future work will be dedicated to a finer rate control of overall rate and redundancy of the mutliply descriptive bitstreams by using a rate-distortion optimization procedure taking into account the channel error or loss characteristics.

\section{REFERENCES}

[1] L. Ozarow, "On a source coding problem with two channels and three receivers," Bell Syst. Tech. J., vol. 59, pp. 19091921, December 1980.

[2] A.A. El Gamal and T.M. Cover, "Achievable rates for multiple descriptions," IEEE Trans. On Information Theory, vol. IT-28, no. 6, pp. 851-857, November 1982.

[3] J.C. Batlo and V.A. Vaishampayan, "Asymptotic performance of multiple description transform codes," IEEE Trans. on Information Theory, vol. 43, no. 2, pp. 703-707, 1997.

[4] M.T. Orchard, Y. Wang, V.A. Vaishampayan, and A.R. Reibman, "Redundancy rate-distortion analysis of multiple description coding using pairwise correlating transforms," Proc. IEEE Intl. Conf. on Image Processing, ICIP'97, vol. I, pp. 608-611, October 1997.

[5] V.K. Goyal, J. Kovacevic, R. Arean, and M. Vetterli, "Multiple description transform coding of images," Proc. IEEE Intl. Conf. on Image Processing, ICIP'98, pp. 674-678, October 1998.

[6] V.A. Vaishampayan, "Design of multiple description scalar quantizers," IEEE Trans. On Information Theory, vol. 39, no. 3, pp. 821-834, May 1993.

[7] V.A. Vaishampayan and J. Domaszewicz, "Design of entropy constrained multiple description scalar quantizers," IEEE Trans. On Information Theory, vol. 40, pp. 245-251, January 1994.

[8] David Taubman, "High performance scalable image compression with ebcot," IEEE trans. on image processing, vol. 9, no. 7, pp. 1158, July 2000.

[9] W. Jiang and A. Ortega, "Multiple description coding via polyphase transform and quantization selection," in Proc. SPIE Intl Conference on Visual Communications and Image Processing, VCIP, January 1999, pp. 998-1008.

[10] S.D. Servetto, K. Ramchandran, V.A. Vaishampayan, and K. Nahrstedt, "Multiple description wavelet based image coding," IEEE Transactions on Image Processing, vol. 9, no. 5, pp. 813-826, May 2000. 